\title{
Strategy in auditory recognition memory
}

\author{
ARTHUR W. TOGA \\ St. Louis University, Departments of Psychology and Neurology, 221 N. Grand Blvd.
}

St. Louis, Missouri 63103

\begin{abstract}
Six male and six female undergraduates were presented with lists of up to six digits in length, each followed by a "probe" digit. They then searched the memorized lists for the presence or absence of the test item. Reaction times (RTs) were used to gain insight into the strategies employed in high-speed recognition scanning. This study showed parallel increases of RTs for both positive and negative responses as a function of list length (LL). However, the serial position of the probe digit produced no significant differences in average RTs. The experiment supports the indication of a serial exhaustive, as opposed to self-terminating search in high-speed scanning for "presence" in immediate memory.
\end{abstract}

The problem of recall from recent memory can be viewed as a search process involving the rapid inspection and testing of those items in store for the critical characteristics necessary for the experimental task. This conceptualization leads to an analytic method of study that is similar to the techniques employed in visual search studies. Usually in visual search, the subject is first presented with a target item and then must search for the presence of that item in a later-presented list.

Implicit in the paradigm of these search process experiments is the supposition that if the selection of the response requires the use of information that is in memory, the latency of the response will reveal something about the process by which the information is retrieved. Fitts and Posner (1967) reported that RTs to locate a probe item increase with the length of the list, whether the search is performed visually or in memory. A second more specific problem in the study of retrieval is the effect of serial position (of the probe) on the latency of response. It has been shown that RT is a function of serial position, whereby searching terminates and the recognition response is made as the target is encountered (Sternberg, 1969).

Two alternative models have been designed to explain the processing necessary for a retrieval task. Townsend (1971) described the two processes that are labeled "serial" and "parallel." By parallel processing, it is meant that the items to be processed are examined concurrently. Although processing begins on all items simultaneously, it does not necessarily end simultaneously. Thus the variance in RT reflects the difference in that completion time. By serial processing, it is meant that the elements are processed one at a time, each being completed before the next is begun. It is necessary to differentiate between two types of serial serach (Sternberg, 1969). In self-terminating serial search, the test stimulus is compared successively to one item in memory after another, either until a match occurs (leading to a positive response) or until all comparisons have been completed without a match (leading to a negative response). In exhaustive serial serach, the test stimulus is compared successively to all of memorized items. Only then is a response made; positive if a match occurs, and negative if otherwise. Since negative responses would always necessitate a fully exhaustive search, the exhaustive model predicts similar RTs for "NO" and "YES" responses. The findings of Sternberg (1966) showed little effect on RT of the serial position of the probe digit in the list. Further evidence for the existence of the exhaustive serial search is given by Wingfield and Branca (1970) and Wingfield and Bolt (1970), with the condition that the search be limited to scanning for the presence of a single target.

The present study was designed to explore some of these issues as well as control and evaluate some of the effects of extraneous variables that may influence the results. In addition to examining the variables of probe position and list length (LL) on reaction time (RT), as Wingfield (1973) did, this study attempts to assess the importance of organismic variables such as sex and environmental influences such as distracting stimuli.

\section{METHOD}

\section{Subjects}

The subjects for this study were six male and six female undergraduates, drawn from an introductory psychology course. Participation in the experiment was voluntary, but the subjects were provided with course credit.

\section{Materials}

A tape consisting of 84 lists of single-digit numbers, 16 different lists for each of six list lengths, was recorded by the experimenter in a clear and distinct manner. The spoken digits were presented at a rate of one digit every $500 \mathrm{msec}$. Each list of each list length was followed by a $1,500-$ msec delay, then by the presentation of a probe digit not exceeding $500 \mathrm{msec}$. The digits for the various lists, as well as the probes, were selected from a table of random numbers, with the constraint that no digit was repeated in any given list. List lengths were one through six digits. The stimulus was made on magnetic recording tape, at a tape speed of $7 \frac{1}{2}(19 \mathrm{~cm})$ ips and were presented on a Sony TC-630 stereo recorder. A quantitative measure of the stimulus was obtained using the Brüel and Kjaer Model 158 audiometer calibrator. The spoken lists of digits were heard at an average level of $76 \mathrm{~dB}$ re $.0002 \mathrm{dyne} / \mathrm{cm}^{2}$, with a range of $72-79 \mathrm{~dB}$. The background noise that was present included music (in 12 of the 14 trials for each LL) and was utilized to 
determine the effect of ambient noise distraction. The average sound pressure level (SPL) for digit presentation in the no-music background (two trials per LL) was $68 \mathrm{~dB}$ and $67 \mathrm{~dB}$ for the music background. The average sound level of the probe digit, which was presented via the opposite recorder channel and thus the opposite ear, was $73 \mathrm{~dB}$ with a range of $70-78 \mathrm{~dB}$.

The occurrence of the probe digit served to activate a Hunter timer (calibrated $.001 \mathrm{sec}$ ) via a photo diode arrangement. The RTs were determined by the subjects' pushbutton response, which also indicated whether the probe digit was perceived as having occurred in the previous list.

Accuracy and consistency for digit presentation times was accomplished by equating presentation time with the length of the tape and the tape speed.

\section{Procedure}

Each subject heard 14 lists for each of the six lengths used. When it was ascertained that the subject fully understood what was required, the experimenter instructed the subject to proceed with six untimed practice lists to insure familiarization with the apparatus and procedure. The timed lists were presented in blocks of 14 lists each, with list lengths of one to six digits. The 14 lists were arranged so that the first list and the last list of a given length contained no extraneous background music. Each trial was separated by a 6-sec delay, and each block of 14 trials was separated by a 12-sec delay: The probe digit appeared randomly in half of the lists for each LL, and when it was present its serial position was varied systematically. The index finger of the preferred hand was used, and the yes and no buttons were changed from the right to the left side for alternating subjects. The order of presentation of the list lengths was increasing for half of the subjects and decreasing for the other half. In addition, the stimulus presentation was randomly varied to the right and left ears.

\section{RESULTS}

The dependent variable for all the analyses was reaction time; and since the population of scores was essentially homogeneous, the means were used in the analysis of the independent variables. Similarly, Wingfield (1973) and others have used the same procedure.

The total error rate was less than $1 \%$ and error analysis showed no effects of major independent variable. The results were calculated with error RTs excluded. Figure 1 shows a positive and visually linear relationship between RT and the number of digits in the mem-

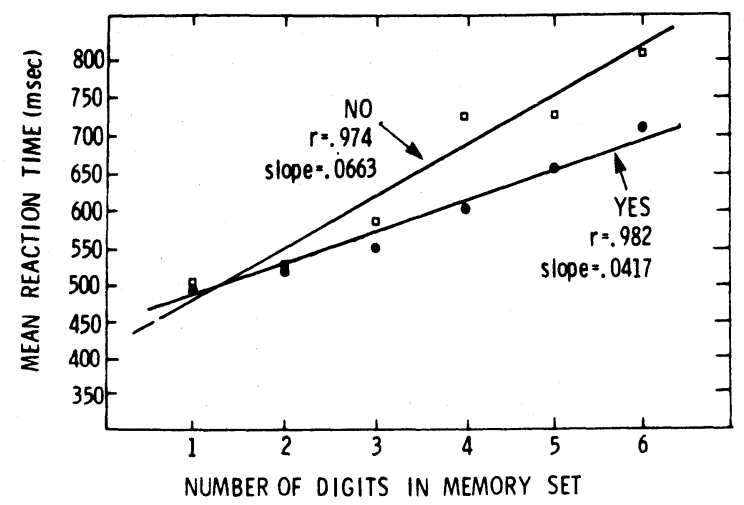

Figure 1. Mean reaction time as a function of list length. ory set. The slopes of the mean RTs for yes and no responses were $41.7 \mathrm{msec} / \mathrm{digit}$ and $66.3 \mathrm{msec} / \mathrm{digit}$, respectively (y-intercept; yes $=437 \mathrm{msec}$, no $=$ $408 \mathrm{msec}$ ). A correlation (Pearson r) between mean RT and the number of digits in the memory set yielded significant coefficients, $\mathrm{r}=.98$ for yes and $\mathrm{r}=.97$ for no. An overall analysis of variance (repeated measurement) showed a significant effect of list length on $R T, F_{(5,55)}$ $=25.40, \mathrm{p}<.001$ but no significant difference between RTs to yes and no responses, $F_{(1,11)}>1$. There was also no significant interaction between $L L$ and response type (yes vs. no). The apparent difference in slope is not significant. In addition, there was no effect on RT from the serial position of the probe in the list.

Both male and female RT functions increased linearly with list length, and the difference between them was significant, $F_{(1,60)}=13.99, p>.001$, with females having greater RTs. The slopes of male and female functions were .056 and .053 with $y$-intercepts of 313 and $532 \mathrm{msec}$, respectively. Finally, there was no difference between the music background trials and the no-music trials.

\section{DISCUSSION}

The results of the present study coincide very closely with those of Wingfield (1973) in terms of the linear relation between mean RT and LL. The procedure used favored almost perfect performance because the design was concerned with the nature of the comparison and not the error rates. A quantitative measure of the extent of linearity, which evidences serial processing (Sternberg, 1966; Townsend, 1971), is assessed by the correlation of the mean RTs and LLs. For both yes and no functions, the correlation was significant. The y-intercept of these latency functions represents the amount of time taken by the motor response, and other unknown processes whose durations are independent of the number of digits in memory.

In Figure 1, the slope of the line represents the mean comparison time per item that the subject needed to decide whether or not the probe digit was a member or the previously presented list of digits. The slope, then, is a pure measure of the time required to process the information, rates of search being limited only by the speed with which the digits are analyzed for the presence of the probe. Sternberg (1966) suggests that the comparison of positive and negative response slopes may give insight into the type of serial search used. The nonsignificant difference of the slopes in the present experiment suggests that the scanning continues through the entire memory set (digit list).

The major implication of this serial exhaustive scan model is that RT for positive responses is relatively unaffected by the serial position of the probe. The results of the present study are consistent with this prediction. The discrepancies between various studies can perhaps be accounted for when considering the mode of presentation; namely that visually presented material produces different results than auditory studies (as suggested by Wingfield, 1973). This quantitative difference can most readily be seen by a comparison of the y-intercepts, when there is a more rapid encoding of the auditory as opposed to the visual probes. This could be explained by a hypothesized acoustic memory store. The absence of a serial position effect is in itself insufficient to support the conclusion of a fully exhaustive, as opposed to a self-terminating, search. However, the finding that the slopes were not significantly longer when the probe item was not present in the list than when it was is more conclusive and adds support to the exhaustive model. 
In an attempt to explain the wide variation of RTs among subjects of a similar population, the experimenter separately evaluated data obtained from male and female subjects. When considering the difference between the male and female subjects, it is important to note the y-intercept. This statistic presumably accounts for those initiating factors in the RT that are independent of LL effects. The difference could be accounted for either by motivational factors or differences in the latency or speed of the basic reflexes.

This study stresses the importance of methodological details and their effects on the results. However, the data presented above suggest that the results are quite robust and that they can be generalized to a wider domain of conditions. For example music has little effect on the RT LL function; and even though there are differences between males and females, it is only in regard to the $y$-intercepts. The slopes (representing search processes) are the same. But it must be noted that this robustness could only be detected with proper methodological changes.

Research has suggested the presence of two kinds of memory search that subjects use in the retrieval of information from short-term memory. One is a high-speed exhaustive scanning process, used to determine the presence of an item in the list, the other is a slow self-terminating scanning process used to determine the location of an item in the list. The assumption is that one must scan a list serially to retrieve information from it, even when it is contained in active memory (Sternberg, 1969). The present study supports this postulate, in that the subject was only required to determine the presence of the probe.

\section{REFERENCES}

Fitts, P. M., \& Posner, M. I. Human performance. Belmont, Calif: Brooks/Cole, 1967.

STERnBERG, S. High-speed scanning in human memory. Science, $1966,9,652-654$.

STERnberg, S. Memory scanning: Mental processes revealed by reaction-time experiments. American Scientist, 1969, 57, 421-457.

Townsend, J. T. A note on the identifiability of parallel and serial processes. Perception and Psychophysics, 1971, 10, 161-163.

Wingfield, A. Effects of serial position and set size in auditory recognition memory. Memory \& Cognition, 1973, 1, 53-55.

Wingfield, A., \& Bolt, R. A. Memory search for multiple targets. Journal of Experimental Psychology, 1970, 85, 45-50.

Wingfield, A., \& Branca, A. A. Strategy in high-speed memory search. Journal of Experimental Psychology, 1970, 83, 63-67.

(Received for publication August 1, 1975.) 